\title{
Médée au Moyen Âge : les interprétations de l'Ovide moralisé
}

\author{
Prunelle DELEVILLE
}

Université Lumière-Lyon 2 et Université de Genève, CIHAM-UMR 5648

\section{Marylène POSSAMAI-PÉREZ}

Université Lumière-Lyon 2, CIHAM-UMR 5648

\begin{abstract}
«Médée au Moyen Âge: les interprétations de l'Ovide moralisé », Figures de la Grèce ancienne, dir. Catherine Gaullier-Bougassas, Turnhout, Brepols, 2020, p. 119-129.
\end{abstract}

Stefania Cerrito, dans une communication prononcée à Lille en $2005^{1}$, a montré que pour ce qui concerne Médée au Moyen Âge, c'est Benoît de Sainte Maure, qui, le premier, fait de la princesse de Colchide un personnage de roman. Mais, ajoute-t-elle, «c'est surtout la magicienne qui intéresse Benoît », pour qui la magie « est la forme suprême du savoir, qui relie l'homme à Dieu ${ }^{2}$ ». Benoît passe sous silence les crimes de Médée. Mais à la fin du XIII et au

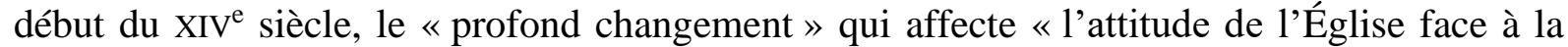
magie » va nuire à la figure de Médée, qui « ne survit que difficilement à la nouvelle atmosphère culturelle ». Seules les mises en prose 2, 3 et 4 du Roman de Troie conservent « ce beau portrait de femme savante et amoureuse ${ }^{3} \gg$.

Trois mouvements distinguent alors les réécritures de la légende de Médée : le premier mouvement, représenté par Prose 1, revient à la Médée cruelle et meurtrière d'Euripide ; le deuxième mouvement dénie à la magicienne tout pouvoir magique : Guido delle Colonne renvoie ces pouvoirs à une erreur païenne. Enfin, un troisième groupe de textes, parmi lesquels la deuxième version de l'Histoire ancienne jusqu'à César, qui intègre Prose 5 et donc la version romane de certaines Hérö̈des d'Ovide, raconte le mythe en entier, depuis la légende de Phrixos et Hellé, origine de la Toison d'or, jusqu'à la disparition définitive de Médée, en passant par l'épisode d'Ypsiphile (qui utilise la $\mathrm{VI}^{\mathrm{e}}$ Hérö̈de), le retour de Jason en Grèce, le rajeunissement d'Éson, la trahison de Jason et la terrible vengeance de Médée qui devient infanticide avant d'être la proie du remords et de mourir noyée. [page 120 éditeur]

${ }^{1}$ S. Cerrito, «Les métamorphoses de Médée au Moyen Âge : analyse du mythe dans les versions françaises, italiennes et espagnoles », Réception et représentation de l'Antiquité, Bien dire et bien aprandre 24 (2005), p. 3956.

\footnotetext{
2 Ibidem, p. 42.

${ }^{3}$ Ibid., p. 44.
} 
L'Ovide moralisé en vers appartient à ce troisième groupe, et ajoute même les aventures de Médée à Athènes, lorsque, ayant fui le royaume de Thessalie, elle épouse Égée et tente d'empoisonner Thésée. L'œuvre de l'anonyme du début du XIV siècle représente ainsi «la synthèse la plus complète et détaillée de l'histoire de la Médée médiévale », puisqu'elle se déploie sur 2000 vers, dans lesquels la Colchidienne est d'abord une princesse « timide et hésitante» «en qui luttent Amour et Raison», puis une «femme amoureuse et (une) magicienne bienfaitrice », enfin « une farouche sorcière ${ }^{4} »$.

Ainsi, les aspects intéressants à développer à propos de l'héroïne de l'Ovide moralisé ne manquent pas, et on a le choix entre la peinture de l'amour naissant et l'étude du magnifique monologue dans lequel l'auteur du XIV ${ }^{\mathrm{e}}$ siècle, traduisant le dilemme de Médée, se montre le digne émule des romanciers du XII ${ }^{\mathrm{e}}$ siècle ; l'examen de la façon dont il traduit, en détail (ce qui est original parmi les adaptations médiévales de la légende de Médée) la procédure suivie par la magicienne pour rajeunir Éson (l'étude du vocabulaire par lequel le translateur rend les différents « charmes » et «enchantements » révélerait sans doute une bonne part d'invention verbale); ou celle d'une autre particularité de l'Ovide moralisé, les interprétations dont il assortit chacune de ses traductions de la fable ovidienne, opérant une deuxième transposition, après le transfert linguistique, la transposition axiologique ${ }^{5}$. C'est cette troisième approche que nous privilégions ici, en particulier parce que la famille de manuscrits que l'une de nous, Prunelle Deleville, édite et étudie est dotée d'interprétations qui lui sont propres ${ }^{6}$.

Nous avons réparti les analyses comme suit: Prunelle Deleville analyse une branche des manuscrits de l'Ovide moralisé tardive, de l'extrême fin du XIV ${ }^{\mathrm{e}}$ siècle jusqu'au début du $\mathrm{XV}^{\mathrm{e}}$ siècle $^{7}$, la branche $Z$, qui présente une réécriture de l'Ovide moralisé ${ }^{8}$. Dans ce remaniement, les allégories sont supprimées ${ }^{9}$, mais des expositions historiques sont ajoutées ou développées et le récit des fables est parfois modifié. Pour le cas de la narration de l'amour naissant entre Jason et Médée et de la conquête de la Toison d'or le remanieur a été très inspiré. Il amplifie largement l'exposition historique de [page 121 éditeur] la fable par l'ajout d'une soixantaine de vers. Une telle configuration ne manque pas de surprendre dans la mesure où cette matière est déjà historique. Elle ne nécessite donc pas, normalement, d'interprétation concrète.

Marylène Possamai interprète ensuite les «moralisations » que présente le plus grand nombre de manuscrits, en s'appuyant sur le texte que Cornelis De Boer a édité au début du XX siècle ${ }^{10}$, essentiellement à partir du manuscrit conservé à la Bibliothèque municipale de Rouen sous la cote O.4. Sont ici prises en compte les moralisations qui concernent la première partie de l'histoire : la conquête de la Toison d'or et le retour de Jason et Médée à Iolcos. Le passage

${ }^{4}$ Ibid., p. 51-52.

${ }^{5}$ G. Genette, Palimpsestes. La littérature au second degré, Paris, 1982, p. 471.

${ }^{6}$ Cette introduction est signée M. Possamai.

${ }^{7}$ Il s'agit des copies suivantes : Paris, BnF, fr. 870 ; Paris, BnF, fr. 19121 ; Paris, BnF, fr. 874 ; Berne, Burgerbibliothek, cod. 10.

${ }^{8}$ Prunelle Deleville édite ce groupe de manuscrits dans sa thèse Métamorphoses des Métamorphoses : étude littéraire et édition critique des manuscrits $Z$ de l'Ovide moralisé, sous la direction de Marylène Possamai et d'Olivier Collet, soutenue à l'Université Lumière Lyon 2 le 13 juin 2019. Cette édition s'appuie sur le manuscrit $Z^{3}$ (Paris, BnF, fr. 870). La référence aux vers de ce texte repose sur la numérotation de cette édition. Quand nous citons l'Ovide moralisé dit « original », nous précisons « éd. C. De Boer».

${ }^{9}$ Dans les témoins $Z^{2}$ (Paris, BnF, fr. 874) et $Z^{1}$ (Berne, Burgerbibliothek, cod. 10), les allégories sont présentes. Selon les études de Richard Trachsler et Laura Endress («Économie et allégorie, notule à propos des manuscrits $Z$ de l'Ovide moralisé », Medioevo Romanzo, 39/2 (2015), p. 350-366), elles auraient été ajoutées dans le modèle de $Z$, à partir d'un représentant de la branche la plus ancienne, qui les possédait.

${ }^{10}$ Ovide moralisé, poème du commencement du quatorzième siècle, éd. C. de Boer, Amsterdam, 1915-1938, $5 \mathrm{t}$. 
étudié occupe les vers 690 à 820 du livre VII ${ }^{11}$. Une rapide analyse permet de bien repérer la hiérarchie établie par le moraliste de l'Ovide moralisé entre la première interprétation, celle par «l'histoire » (de type évhémériste), qui constitue à la fois un «nettoyage de la fable » (elle supprime les aspects invraisemblables pour la raison ou inacceptables pour la morale et fournit à la fable un sens littéral acceptable) et un relais sensible, un tremplin vers les vérités intelligibles, et celle que l'auteur appelle seule "allégorie», une interprétation par la signification « intelligible » justement, spirituelle, et, en l'occurrence, chrétienne : il s'agit de l'un des trois sens spirituels que les Pères de l'Église attribuaient aux seules Écritures saintes, ici le sens typologique, l'allégorie au sens restreint, le quid credas conforme au dogme de l'Église. Conformément à l'ordre établi par l'auteur de l'Ovide moralisé, nous évoquerons d'abord le niveau « sensible » de l'interprétation, dans l'œuvre « originale ${ }^{12}$ » et dans la version remaniée, avant d'examiner le niveau spirituel.

Dans la version originale, le récit de la conquête de la Toison d'or reçoit un premier niveau d'interprétation historique. Logiquement, la légende des Argonautes ne nécessite pas d'explication concrète: elle a un sens littéral acceptable, puisqu'elle est vraie dans la conception médiévale. Cependant, le premier auteur de l'Ovide moralisé émet une restriction, lorsqu'il écrit que « Toute ceste fable est histoire / Et de Pelye et de Jason / Fors seulement de la toison ${ }^{13} \gg$ (éd. C. De Boer, v. 690-692). Seule la Toison d'or reste donc à interpréter. L'exégète en fait l'équivalent de Médée enfermée par son père, que personne n'aurait pu ravir sans le conseil de la princesse elle-même. L'exposition retrouve le schéma folklorique du conte merveilleux.

Le remanieur amplifie largement l'exposition en complétant cette trame. La nouvelle lecture qu'il fournit ressemble ainsi complètement à un conte. Le décor devient plus précis : Médée n'est plus seulement enfermée on ne sait où, mais elle est retenue dans un château, dans une tour rendue inaccessible par la présence d'un enchantement. Le père est présenté comme un jaloux, un sot. Il promet à celui [page 122 éditeur] qui réussira à délivrer sa fille un grand trésor et de belles noces. Sachant qu'il est impossible par des forces humaines d'arriver à un tel exploit, le père conserve ainsi tout pouvoir sur son enfant. De nombreux chevaliers se présentent pour accomplir cette prouesse, qui tous meurent. Un jour arrive un jeune homme, qui figure Jason. Médée tombe amoureuse de lui, et mue par la pitié qu'elle ressent pour ce vaillant garçon, décide de l'aider à défaire l'enchantement. Le canevas de cette interprétation n'évacue pas totalement, a priori, le merveilleux en reprenant à son compte un schéma et des motifs du conte merveilleux : le père possessif qui ne veut donner la main de la princesse qu'en soumettant les prétendants à une épreuve inhumaine et l'aide magique que reçoit le héros qui s'est qualifié ${ }^{14}$. Pourtant, nous relevons dans le même temps un effort d'explication rationnelle de la fable. Dans le récit de cette fable, le remanieur avait qualifié la Toison d'or de «droite faerie » (v. 18, « authentique sortilège »). Dans l'interprétation, il n'emploie plus ce vocabulaire qui dénote l'univers merveilleux. Au contraire, il a plusieurs fois recours au mot « enchantement 》 (v. 745, v. 748, v. 811), une fois au syntagme «art magique » (v. 746) qui renvoie à la maîtrise d'une technique et non à la possession d'un pouvoir surnaturel. Cet aspect rejoint ce qu'évoque L. Harf-Lancner à propos de la rationalisation du merveilleux dans le Lancelot en prose :

11 Édition C. De Boer, t. 3, p. 31 à 34.

12 Prunelle Deleville, qui reprend ici la plume, utilise l'adjectif « original» pour opposer la version de l'Ovide moralisé du début du XIV $\mathrm{XI}^{\mathrm{e}}$ siècle à sa réécriture. Elle différencie aussi l'auteur « original», ou le « premier» auteur, de son « remanieur » ou « l'adaptateur » de la famille $Z$.

13 «Tout le contenu de ce récit fabuleux est historique, pour ce qui concerne Pélias et Jason, à l'exception de la Toison. » Toutes les traductions de l'Ovide moralisé sont de notre fait.

${ }^{14} \mathrm{C}$ 'est ce schéma que suit, dans sa première partie, le lai de Marie de France intitulé Les deux amants. Voir Marie de France, Lais, dans Lais bretons (XII ${ }^{e}$ XIII $^{e}$ siècles) : Marie de France et ses contemporains, éd. et trad. N. Koble et M. Séguy, Paris, 2018, p. 388-407. 
Dans cette vision rationalisée, les attributs de la fée ne sont pas attachés à sa nature fantastique mais acquis par le fruit d'un savoir et inscrits dans le temps [...]. Les interventions féeriques sont qualifiées d'enchantements et le vocabulaire de la féerie se confond avec celui de la sorcellerie ${ }^{15}$.

Le recours à des éléments a priori magiques et la reprise de thématiques du conte merveilleux correspondent donc paradoxalement à une entreprise de rationalisation du récit.

Cependant, cet effort de rationalisation n'est pas sans difficultés, ce qu'exprime implicitement le remanieur. Tout d'abord, dans les quatre manuscrits qui présentent cette exposition, aucune rubrique n'indique de démarcation entre la fable et l'exposition, comme c'est toujours le cas dans le reste du texte. Cette incongruité pourrait être signifiante et s'analyser comme le statut ambigu de ce passage, comme la marque de la porosité de la frontière entre fiction et vérité, lorsqu'il s'agit de traiter de la Toison d'or. L'adaptateur de la famille $Z$ dresse lui-même ce constat implicite à propos du caractère insaisissable du sujet de la fable, à la fin de l'exposition, au moment où Médée délivre le château du sortilège :

Tant fist et tellement ovra,

Par force de sens et d'avoir

- Car moult estoit de grant savoir -

Qu'elle [deffist] l'enchantement. [page 123 éditeur]

Ce ce conte, si ne me ment,

Siques la toison doree

Est ainssi prisse pour Medee. (v. 808-814)

[Elle fit tant et œuvra tellement, par la vertu de son intelligence et de ses biens - car elle possédait un grand savoir - qu'elle (annula) l'enchantement. C'est ce que raconte cette histoire, si elle ne me trompe pas. C'est ainsi que la Toison dorée est comprise comme signifiant Médée.]

L'emploi de la subordonnée consécutive «fist tant que » marque une volonté d'expliciter la fable, déjà exprimée par la mise en valeur du «sens» et du «grant savoir » de Médée. L'intelligence et non la jouissance de pouvoirs surnaturels est la cause de la conquête de Jason. Cependant, la rime entre «enchantement » et l'hypothétique «si ne me ment » peut traduire qu'une part de mystère reste irréductible, impénétrable par la raison. Le remanieur de $Z$ affirme d'ailleurs, dans la présentation de son exposition, que « qui au droit san veut tendre / ainssi doit la fable entendre » (v. 733-734, « celui qui veut s'approcher de l'exacte signification de la fable / doit la comprendre de cette façon »). L'emploi du verbe « tendre » invite à penser qu'une part du récit échappe à la vérité, « au droit sans », ce qui suggère le caractère insaisissable de cette fable. En outre, le remanieur n'emploie nulle part ailleurs le verbe « tendre » pour désigner son effort de rationalisation. Il est toujours beaucoup plus sûr de lui. Ainsi, l'opposition entre l'expression d'une réserve ( « tendre ») et celle d'une forte adhésion ( « doit la fable entendre ») exprime la difficulté d'interpréter cette matière d'un point de vue concret. Le remanieur paraît dire à demi-mots que quelque chose échappe à la raison dans la fable, comme lorsqu'il énonce au tout début de l'exposition que :

Ceste fable est vraye ystoire

Fors seullement de la toison,

Conbien que grant mencion

En est faite en mains ystoires

Qui sont approuvez pour voires. (v. 726-730)

[Cette fable est de l'histoire vraie, sauf seulement pour la Toison, bien qu'elle soit largement mentionnée dans de nombreuses histoires qui sont considérées comme vraies.]

${ }^{15}$ Laurence Harf-Lancner, Les fées au Moyen Âge, Paris, 1984, p. 413. 
Les deux premiers vers reprennent les termes du premier auteur. Dans un second temps, la concession ajoutée, introduite par «conbien que», signale que le propos échappe à la normalisation et à la vérité de l'histoire de Médée pourtant affirmée par la rime entre « ystoires » et « voires ». Une tension semble donc se manifester, qui traduit la difficulté de décrypter cet épisode d'un point de vue rationnel. Un tel embarras a certainement porté le remanieur à tenter d'expliquer plus longuement la part de surnaturel contenue dans la conquête de la Toison d'or, alors qu'il s'agit pourtant d'une matière historique sur laquelle il ne convient normalement pas de s'appesantir dans l'explication évhémériste. N'y a-t-il pas aussi dans ce traitement particulier du mythe l'expression d'une part de fascination ? Selon Richard Buxton, [page 124 éditeur] qui a traité des pouvoirs de Médée, la fascination qu'exerce la magie de Médée tient justement au fait que «c'est précisément parce que l'acte d'enchantement a une base prétendument 'rationnelle' que la magie est encore plus convaincante ${ }^{16}{ }$.

Ainsi, grâce à l'expression d'une tension entre ce qui est vrai et ce qui échappe à la vérité, le nouvel auteur réussit à saisir et à rendre la fascination liée au statut ambigu de l'épisode de la Toison d'or. Par l'union implicite entre vérité historique et topoï littéraires merveilleux, il capte la vérité de la fable.

L'auteur original ${ }^{17}$ de l'Ovide moralisé n'a pas la même vision des choses, puisque pour lui l'exposition historique n'est qu'un relais sensible qui ouvre vers le sens spirituel, ce sens « plus haut », « meilleur » selon lui. « Or vous dirai l'alegorie ${ }^{18} »:$ c'est la formule par laquelle le moraliste marque la hiérarchie entre le sens «littéral», concret, sensible (naturaliste ou évhémériste), et le sens spirituel (qui peut être typologique - quid credas -, tropologique - quid agas -, et/ou anagogique - quo tendas ${ }^{19}$ ).

C'est ici le sens typologique : Pélias est le diable, qui, par jalousie, envoya l'humain lignage cueillir le fruit de la science (c'est-à-dire dans la fable les Argonautes à la conquête de la Toison d'or - mais ce n'est pas explicité : nous sommes au livre VII, l'auteur considère ses lecteurs comme avertis). De même il faut comprendre que le Christ est l'interprétation de Jason, qui dans sa sagesse voulut, pour sauver l'homme, s'unir à « la vierge honoree / Pour avoir la toison doree, / C'est la sainte virginité / De la mere » (v. 731-744, « à la Vierge honorée, pour acquérir la Toison d'or, c'est-à-dire la sainte virginité de la mère »). Cependant les choses ne sont pas claires, et les deux personnages de Jason et de Médée ne sont pas bien distingués, car ce n'est pas Jason, mais Médée qui « vit la dolour et la paine / Et la mort que home atendoit » (v. 734-735, «vit la douleur et la peine, et la mort qui attendait l'homme »), qui en eut «pitié grant et conpascience » (v. 737-738, « une grande pitié et une vive compassion »), et qui « dona l'erbe et la racine / De la salvable medecine» (v. 747-748, «donna l'herbe et la racine qui composaient le remède salutaire $»)$. En fait c'est l'enchantement préparé par la magicienne qui reçoit l'équivalence, point par point, de l'Incarnation. Le magicum est interprété par le miraculum $^{20}$, la seule magie admise par la pensée chrétienne est le miracle de l'Incarnation divine : nous avons ici une allégorie énumérative ou descriptive ${ }^{21}$. L'herbe donnée par Médée a été cueillie «dou ventre à la vierge Marie» (v. 750 ; la terre cultivée par Dieu est le [page

${ }^{16} \mathrm{R}$. Buxton, « Les yeux de Médée : le regard et la magie dans les Argonautiques d'Apollonios de Rhodes », dans La magie dans l'Antiquité tardive. Les mythes, éd. A. Moreau et J.-C. Turpin, Montpellier, 2000, p. 267.

${ }^{17}$ C'est Marylène Possamai qui aborde ici l'étude de la version « originale ».

18 «Je vais maintenant vous dire l'allégorie. ».

${ }^{19}$ Quid credas : « ce qu'il faut croire»; quid agas : « ce qu'il faut faire »; quo tendas : « ce vers quoi il faut tendre ». $C f$. H. de Lubac, «Sur un vieux distique. La doctrine du ' quadruple sens ' », dans Mélanges Ferdinand Cavallera, Toulouse, 1948, p. 347-366.

${ }^{20}$ J. Le Goff, « Le merveilleux dans l'Occident médiéval », dans L'étrange et le merveilleux dans l'Islam médiéval, éd. Jeune Afrique, Paris, 1978, p. 61-115, repris dans L'imaginaire médiéval, Paris, 1985, rééd. 1991, p. 17-39: magicum: «magique», miraculum : « miraculeux ».

${ }^{21}$ A. Strubel, « Grant senefiance $a$ » : Allégorie et littérature au Moyen Âge, Paris, 2002, p. 43. 
125 éditeur] ventre de la Vierge, v. 751-753), et la préparation reçoit l'équivalent de la passion : elle fut en effet «ou mortier de la crois triblee / Et de vinaigre destrempee » (v. 755-756, « écrasée au mortier de la Croix et délayée avec du vinaigre »).

À cette époque tout est prétexte à l'interprétation par les instruments de la Passion, particulièrement la Croix, qui devient objet de dévotion à partir du XIII ${ }^{\mathrm{e}}$ siècle. L'enchantement, le charme, c'est « la chars » du Christ (il y a peut-être un jeu sur les sonorités de charme et de char : «La chars fu l'erbe et la poison / Qui trait le siecle a garison », v. 757-758, «La chair était la plante et la potion qui mène le monde à sa guérison »). Ce n'est pas exactement la conquête de la Toison d'or, mais plutôt la préparation magique de Médée, qui est assimilée à la Passion : «Li jus qui de cele herbe issi / Fu le sans et l'iaue autresi / Qui dou cors Jhesucrist raierent » (v. 759-761, « Le suc qui sortit de cette plante était le sang et l'eau qui coulèrent du corps de Jésus Christ »). Dans l'Ovide moralisé, un morceau de bois figure souvent la Croix et un liquide est souvent la représentation du sang du Christ !

Bien sûr le dragon gardien de la Toison est le diable (v. 766-770); les taureaux sont les compagnons du diable et le feu qui sort de leur gueule figure les mauvaises paroles (le péché de langue fustigé par saint Louis et théorisé par Raoul Ardent ${ }^{22}$ ) : «Feu de venimeuse reprouce / D'affis et de derrisions / Et de falses distrucions » (v. 772-774, « Le feu du reproche venimeux, des défis et des moqueries, des mensonges destructeurs »). On sait combien le moraliste fustige ce peccatum linguae. La fuite des Minéens est celle des disciples au Jardin des Oliviers (v. 775776). Les dents du dragon semées par Jason représentent « la sainte semance / De la crestienne creance » (v. 783-784, « la sainte semence de la foi chrétienne »). Les chevaliers qui en sortent sont les disciples du Christ qui, armés de justice (v. 792) se battirent contre « les anemis de la foi » (v. 795, « les ennemis de la foi »). Les choses sont claires aux vers 799-800: Jason est le Christ, la Toison est la Vierge ${ }^{23}$. Même le démembrement de son frère par Médée figure la Passion du Christ (v. 807-813).

La question qui se pose à propos de ces deux allégories est celle de leurs sources possibles. On sait que l'anonyme travaillait sur un manuscrit glosé des Métamorphoses $^{24}$ : les gloses marginales de ce type de manuscrit reprennent souvent des commentaires antérieurs comme les Integumenta de Jean de Garlande ou les Allegoriae d'Arnoul d'Orléans, dont Frank T. Coulson a bien montré qu'ils étaient souvent recopiés de façon indifférenciée dans les gloses marginales des manuscrits [page 126 éditeur] des Métamorphoses, à partir du XII ${ }^{\mathrm{e}}$ siècle $^{25}$. L'un de ces manuscrits, le manuscrit du Vatican, Biblioteca Apostolica Vaticana, Vat. Lat. 1479, pourrait être assez proche de celui-ci qu'utilisait l'anonyme du début du XIV siècle. Or la glose marginale de ce manuscrit n'interprète Médée que d'un point de vue « évhémériste », ou historique, en faisant de la magicienne l'incarnation de la sagesse, de la

${ }^{22}$ Raoul Ardent, Speculum universale, cité par Carla Casagrande et Silvana Vecchio, Les péchés de la langue, Paris, 1991, p. 174.

${ }^{23}$ Remarquons la cohérence de ces différentes lectures : l'interprétation historique a dit que la Toison était Médée. Jason est le Christ, « doux mouton» qui «fu couvert» (v. 803) de la toison (qui « s'aombra » dans le ventre de la Vierge, dit ailleurs le texte : cf. par exemple II, 4513, IV, 6576, XII, 1951).

${ }^{24}$ M. Possamai-Pérez, «L'Ovide moralisé, ou la 'bonne glose' des Métamorphoses d'Ovide », Regards croisés sur la glose, Cahiers d'Études Hispaniques Médiévales, 38 (2008), p. 181-206.

${ }^{25}$ Voir F. T. Coulson, «Ovid's Metamorphoses in the School Tradition of France, 1180-1400: Texts, Manuscript Traditions, Manuscript Settings », dans Ovid in the Middle Ages, éd. J. G. Clark, F. T. Coulson et K. L. McKinley, Cambridge, 2011, p. 48-82. Tout récemment encore, F. Ploton-Nicollet (« Lire Ovide à la fin du Moyen Âge : un ensemble de scholies métriques en marge du texte des Métamorphoses dans le manuscrit Saint-Omer, Bibl. mun. 678 », dans La rigueur et la passion, Mélanges en l'honneur de Pascale Bourgain, éd. C. Giraud et D. Poirel, Turnhout, 2016, p. 565-579), a pu constater que le manuscrit de Saint-Omer, Bibliothèque municipale, 678, daté du dernier tiers du XIII ${ }^{\mathrm{e}}$ siècle, qui recopie les sept premiers livres des Métamorphoses, les accompagne de commentaires marginaux qui parfois « sont des citations des deux grands commentaires allégoriques et versifiés des Métamorphoses », celui de Jean de Garlande et celui de Giovanni del Virgilio (art. cit., p. 567-568). 
science : la glose, qui se présente comme une allégorie énumérative, n'est pas la source de l'interprétation de Médée dans la version «commune » de l'Ovide moralisé ${ }^{26}$.

Le Commentaire Vulgate étudié par F. T. Coulson propose une lecture morale selon laquelle Jason et sa conquête de la Toison d'or représentent les marchands dangereux et où Médée est la fallacia, la supercherie, la tricherie : il n'y a pas non plus de rapport avec notre texte. Dans les commentaires un peu plus tardifs que l'Ovide moralisé, les Allegoriae de Giovanni del Virgilio $^{27}$ et le commentaire du manuscrit du Vatican, Biblioteca Apostolica Vaticana, Vat. lat. 2877 ( ca 1350, édité par F. T. Coulson ${ }^{28}$ ), l'interprétation est liée à l'argent qui est cause de conflits, au tribut, à la Toison d'or comme un trésor. Elle évoque aussi la lecture de Jason comme marchand du Commentaire Vulgate.

Pour ce qui est de l'allégorie typologique, c'est encore plus complexe. L'auteur de l'Ovide moralisé l'a-t-il lue dans l'un des ouvrages qu'il connaissait et qu'il utilisait pour son travail ? Nous n'en avons pas trouvé la trace.

Pierre Bersuire, le bénédictin qui accomplit le même travail que notre anonyme, au même moment, mais en prose et en latin, une première fois sans connaître l'Ovide moralisé, une deuxième fois après l'avoir eu sous les yeux, propose l'interprétation [page 127 éditeur] suivante : «Per vellus aureum possumus intelligere divitias temporales et maxime divitias ecclesiae $^{29}$. » Jason est le bon prélat qui veut acquérir ces richesses. Les taureaux sont les cruels tyrans, et le dragon est le diable. Le roi Aeétès est Dieu le Père, Médée sa fille est « virginem gloriosam, vel etiam sapientiam ${ }^{30} »$ et Jason veut devenir familier avec la fille du roi, c'est-àdire la bienheureuse Vierge ou la sagesse. Le joug qui doit soumettre les taureaux furieux est celui de l'enseignement (« jugum disciplinae »), et la pénitence par laquelle il faut labourer la terre de leur cœur ( « arare eos cogat per poenitentiam terram cordis suis ») ; «Item ad ipsum pertinet draconem id est diabolum superare », "c'est par cette même pénitence qu'il faut soumettre le dragon, c'est-à-dire le diable, «et dentes ejus id est temptationes et morsus praedicando seminare », « et il faut semer ses dents, c'est-à-dire les tentations et les morsures grâce à la prédication. ».

Les soldats sont les hommes bons et humbles, qui combattent spirituellement l'un contre l'autre et tuent la vie charnelle :

Vel dic quod Jason est Christus qui assumpta uxore Medea id est nostra humanitate, boves id est tyrannos jugo fidei subire coegit [...] draconem diabolum superavit, dentes ejus id est peccatores in ecclesia seminatos per fidem [...] quorum tamen instigante diabolo alter contra alterum per detractionem et invidiam nunc insurgit.

${ }^{26}$ C'est pourtant l'interprétation la plus courante au Moyen Âge : ainsi, c'est celle qu'on peut lire dans la General Estoria d'Alphonse X, comme l'a récemment montré I. Salvo-Garcia, «Mujeres sabias en la historiografía alfonsí : la infanta Medea », dans Histoires, Femmes, Pouvoirs. Péninsule Ibérique (IXe-XVe siècle). Mélanges offerts au Professeur Georges Martin, Paris, 2018, p. 339-365. I. Salvo-Garcia, étudiant « l'infante Médée », c'est-à-dire la bonne fille du roi, rappelle que pour Alphonse X, la sagesse (le savoir, et en particulier le trivium, mais aussi l'astrologie) aussi bien que la magie sont fondamentales, chez un homme autant que chez une femme.

27 Qui datent de 1322 et ont été éditées aussi par Fausto Ghisalberti, « Giovanni del Virgilio espositore delle Metamorfosi », Giornale dantesco, 34 (1933), p. 1-110.

28 Frank T. Coulson, «Vaticanus latinus 2877: A Hitherto Unedited Allegorization of Ovid's Metamorphoses », The Journal of Medieval Latin, 2 (1992), p. 1-61.

${ }^{29}$ Petrus Berchorius, Reductorium morale, XV, 2-15 : Ovidius moralizatus naar de Parijse druk van 1509 : Metamorphosis Ouidiana Moraliter a Magistro Thoma Walleys Anglico de professione praedicatorum sub sanctissimo patre Dominico : explanata Venundatur in aedibus Ascensianis et sub pelicano in vico sancti Iacob Parisiis, éd. J. Engels, Utrecht, 1962, p. 109-110 (toutes les traductions de Bersuire sont nôtres) : « Par la Toison d'or nous pouvons comprendre les richesses temporelles et particulièrement les richesses de l'Église. »

30 Ibidem, p. 110 : « la Vierge glorieuse, ou aussi la sagesse. » 
[Ou alors Jason est le Christ qui a épousé Médée, c'est-à-dire notre humanité. Il a dompté les taureaux c'est-à-dire les tyrans sous le joug de la foi [...], il a triomphé du dragon le diable: ses dents sont les pécheurs semés dans l'église par la foi [...], mais qui se battent l'un contre l'autre à l'instigation du diable, sous l'effet de la diffamation et de la haine.]

Erat Medea filia Aetae mirabilis incantatrix, sicut ponit Ovidius. Ipsa lucem in tenebras subito vertebat, ventos inducebat et pluvias choruscationes et grandines et terrae motus. Et flumina retroire faciebat et arbores florere et senes ad juventutis gloriam revocabat. Haec etiam quendam currum habebat quem duo dracones portabant, et eam ad colligendas herbas, et ad speculandum sydera quolibet deferebant. Ista igitue amore Jasonis victa, et patriam et regnum cujus erat haeres reliquit et in Thessaliam cum eodem Jasone fugit. Patris etiam divitias secum tulit. Istud potest dici contra maliciam mulierum, quae arte mirabili sciunt homines incantare, in tantum quod suis incantationibus generant tenebras ignorantiae, ventos superbiae, choruscationes concupiscentiae vel contumeliae, in tantum quod ipsos faciunt per curiositatem florere, et si sint senes faciunt eos ad juventutis lasciviam pervenire. Dracones id est daemones currum suae levis voluntatis agitant, et per instabilem vagabilitatem undique eas portant. Istae amore fatuorum juvenum [page 128 éditeur] quandoque patrem et patriam deserunt, paternis thesauris ereptis haeredidate et verecundia post positis cum eis vadunt ${ }^{31}[\ldots]$.

[Médée, la fille d'Aeétès, était une enchanteresse merveilleuse, comme le dit Ovide : elle changeait subitement la lumière en ténèbres, elle commandait aux vents, aux pluies et aux éclairs, au tonnerre, aux tremblements de terre, elle inversait le cours des fleuves et elle faisait fleurir les arbres. Elle ramenait les vieillards à la gloire de la jeunesse. Elle avait un char conduit par deux dragons qui la portaient où elle voulait pour aller récolter ses herbes et regarder les étoiles. Elle fut donc vaincue par l'amour de Jason : elle abandonna sa patrie et le royaume dont elle était l'héritière et s'enfuit avec Jason en Thessalie; elle emporta même avec elle les richesses de son père. Voici ce qu'on peut dire contre la malice des mauvaises femmes : elles savent enchanter les hommes par leur art merveilleux, au point que par leurs enchantements elles engendrent les ténèbres de l'ignorance, les vents de l'orgueil, les éclairs de la concupiscence ou des injures ; au point qu'elles les font fleurir de curiosité, et s'ils sont vieux elles les font revenir au libertinage de la jeunesse. Les dragons, c'est-à-dire les démons, soulèvent le char de leur volonté frivole et les emportent partout dans des errances instables. Elles abandonnent leur père et leur patrie pour l'amour de jeunes sots, et s'en vont avec eux après avoir emporté les trésors paternels et renoncé à leur héritage et à leur pudeur [...].]

L'allégorie de Pierre Bersuire, on le voit, est morale, et ne reprend pas l'interprétation christianisante de l'Ovide moralisé : même s'il a eu sous les yeux cette interprétation, il se peut qu'il l'ait trouvé soit trop complexe, soit à la limite de l'hérésie, à cause de cette transformation du magicus en miraculus, cette explication des « enchantements et des charmes » de Médée par l'Incarnation et la Passion du Christ.

Ainsi donc, en l'état actuel de nos recherches, nous n'avons pas trouvé la trace de cette interprétation, ni chez les prédécesseurs de l'auteur du poème du XIV ${ }^{\mathrm{e}}$ siècle, ni chez ceux qui ont pu le lire et s'inspirer de lui. Il se peut bien sûr que des textes importants nous aient échappé, aussi c'est avec prudence que nous émettons l'hypothèse que cette allégorie est le fait de notre poète roman ${ }^{32}$.

${ }^{31}$ Voir ibidem, p. 110-111.

${ }^{32}$ Cette hypothèse semble la plus probable, et nous remercions Jean-Yves Tilliette de l'avoir confirmée le jour du colloque. 
Nous supposons d'autre part ${ }^{33}$ que l'interprétation historique de l'auteur original de l'Ovide moralisé a été influencée par le Roman de Troie ${ }^{34}$. Au début de sa nouvelle exposition, le remanieur de la famille $Z$ évoque «l'ystoire de Troye » (v. 731) qui réfère au Roman de Troie. Il la désigne certainement car il a reconnu la source du premier auteur de l'Ovide moralisé pour [page 129 éditeur] ce passage. Il emprunte lui aussi à ce texte ${ }^{35}$. D'autres éléments qu'on trouve au livre IV de l'Ovide moralisé original ont pu également lui venir en mémoire ${ }^{36}$. Quoi qu'il en soit, ce développement est la marque du charme qu'exercent la figure de Médée et les éléments «merveilleux » liés à la Toison d'or et aux «enchantements » de la princesse de Colchide.

33 C'est ici la conclusion de Prunelle Deleville.

${ }^{34}$ Sur ce point, nous renvoyons à notre thèse dans laquelle nous démontrons que l'auteur original s'inspire du Roman de Troie, v. 763-770 (Le Roman de Troie, éd. E. Baumgartner et F. Vielliard, Paris, 1998).

${ }^{35}$ Nous pensons notamment aux vers sur la façon dont est gardée la Toison d'or (Roman de Troie, éd. cit., v. 1349-1351 et v. 1787-1789).

36 Jean-Yves Tilliette, dans la conversation qui a suivi cette communication, proposait de faire un lien entre l'interprétation remaniée de la Toison d'or et un passage concernant Persée (éd. C. De Boer, IV, v. 6246-6264). Il est effectivement question dans la fable de Persée et d'Atlas de la richesse que ce dernier tente de protéger en enfermant la pomme dorée dans un jardin, gardée par un serpent. Le remanieur aurait pu broder l'explication à partir de ce passage. Nous remercions encore Jean-Yves Tilliette pour cette piste. Nous pensons aussi à la fable de Danaé retenue prisonnière par son père (éd. C. De Boer, IV, v. 5410-5437). 